\title{
Simultaneous particles influence on the LAGO's Water Cherenkov Detectors signals
}

\section{Otiniano, ${ }^{a, *}$ M. Suarez-Duran, ${ }^{b, c}$ Christian Sarmiento-Cano, ${ }^{d} \mathbf{H}$. Asorey ${ }^{d}$ and I.} Sidelnik ${ }^{e}$ on behalf of the LAGO Collaboration

(a complete list of authors can be found at the end of the proceedings)

${ }^{a}$ Comisión Nacional de Investigación y Desarrollo Aeroespacial, CONIDA, Lima, Perú

${ }^{b}$ Universidad de Pamplona, Departamento de Física y Geología

Pamplona, Colombia

${ }^{c}$ Université Libre de Bruxelles

Brussels, Belgium.

${ }^{d}$ Instituto de Tecnologías en Detección y Astropartículas (ITeDA, CNEA/CONICET/UNSAM)

Centro Atómico Constituyentes, Av. General Paz 1499, 1450 Villa Maipú, Buenos Aires, Argentina

${ }^{e}$ Departamento de Física de Neutrones, Centro Atómico Bariloche (CNEA/CONICET)

Av. Bustillo 9500, 8400 San Carlos de Bariloche, Argentina

E-mail: lotiniano@conida.gob.pe

The Latin American Giant Observatory (LAGO), operates an extensive network of Water Cherenkov Detectors (WCD) by a non-centralized and collaborative network of Universities and Research Institutes in Iberoamerica. To estimate the charge distribution produced by secondaries particles interacting with WCDs, LAGO developed a simulation framework (ARTI). ARTI comprises a chain of simulations that starts with the well known primary flux of galactic cosmic rays that reach Earth and finish estimating the expected WCDs signals at any site on ground.

Based on the first stage of ARTI, that uses COSIKA to simulate the expected flux of secondaries that reach LAGO sites, we re-analyze this flux searching for simultaneous particles reaching the detectors. We perform a spatial analysis of CORSIKA's simulated air showers in the field of view of four typical WCD in extreme sites of the LAGO network and in time windows of the electronic acquisition system.

We have found that simultaneous particles reaching the WCD modify the deposited energy distribution into the detector even for low energy range and low altitude sites, compared with the previous single-particle approach. This result impacts the WCD's calibration and could play an important role in discriminating primaries and defining observables for GRBs detection at high altitude LAGO sites.

$37^{\text {th }}$ International Cosmic Ray Conference (ICRC 2021)

July 12 th - 23rd, 2021

Online - Berlin, Germany

\footnotetext{
${ }^{*}$ Presenter
} 


\section{Introduction}

The LAGO Collaboration is a non-centralized and distributed network of more than 100 scientists from 10 institutions, 9 in Latin America: Argentina, Bolivia, Brazil, Chile, Colombia, Ecuador, Guatemala, Mexico, Peru, and Spain. LAGO is oriented to basic research on three branches of Astroparticle physics: the Extreme Universe, Space Weather phenomena, and Atmospheric Radiation at ground level.

LAGO operates a detection network of water Cherenkov detectors (WCD) spanning over different sites that are located at significantly different latitudes and different altitudes (from level from the sea to more than 5000 meters above sea level). The network goes from Mexico (Sierra Negra) to Antarctica, in this way we cover a wide range of geomagnetic rigidity cut off values and atmospheric absorption levels [1,2].

In order to carry out the proposed scientific studies, LAGO has built and implemented a framework of computational models called ARTI. These models make uses of different programs starting with the propagation of the primary cosmic ray through the interstellar medium near the Earth, and ends in the response of the WCD to the secondary particles that propagates in the atmpsphere. ARTI is unique, since allows to estimate the flux of secondary cosmic rays at ground level anywhere on Earth using the local coordinates and the rigidity cut off at the site [2,3].

All LAGO studies exploit the single particle technique (SPT) by looking for significant excesses in the background signal count at different sites on different time scales. For example, minutes and milliseconds for searching GRBs, hours for searching Forbush decreases. Because of this, a profound understanding of the interaction of secondary cosmic ray particles with the WCD result of the utmost importance.

In this article we detail some ARTI characteristics and then propose a correction due to the entry of simultaneous particles (multiparticles) in the LAGO detectors, which has not been taken into account yet in this framework.

\section{Extensive Air Showers Modeling}

The flux of secondary particles has been estimated following the method implemented by LAGO for its space weather program [2-4]. This method estimates the galactic cosmic ray flux $(\Phi)$ at an altitude of $112 \mathrm{~km}$ a.s.l., in accordance with the Linsley atmospheric model [5], i.e.

$$
\Phi_{(i)}\left(E_{P}, Z, A, \Omega\right) \simeq j_{0}(Z, A)\left(\frac{E_{p}}{E_{0}}\right)^{\alpha(Z, A)} .
$$

Here, the index $i$ indicates the kind of geomagnetic field correction applied, i.e. the definition of the rigidity cutoff, meanwhile the spectral index is consided as a constant with respect to the energy from $10^{11} \mathrm{eV}$ to $10^{15} \mathrm{eV}$ [6], the energy reference $E_{0}=10^{12} \mathrm{eV}$, and each type of GCR considered is individualized by its mass number (A) and atomic number (Z). The values for $\alpha$ and the normalization parameter $j_{0}$ are taken from the compilation in [7]. In this work, we have calculated $\Phi_{(i)}$ using the standard definition of the rigidity cutoff $\left(\Phi_{(0)}, \Phi\right.$ hereafter), during one hour of flux, and primaries in the range $1 \leq \mathrm{Z} \leq 26$, for $\theta$ from $0^{\circ}$ to $90^{\circ}$. More details see [4]. The development of the EAS have been computing using CORSIKA [8], version 75600, compiled 
using the following options: QGSJET-II-04 (Ostapchenko, 2011); GHEISHA-2002; EGS4; curved and external atmosphere, and volumetric detector). These two computing, $\Phi$ and EAS-CORSIKA, have been runned throught the LAGO's ARTI tool.

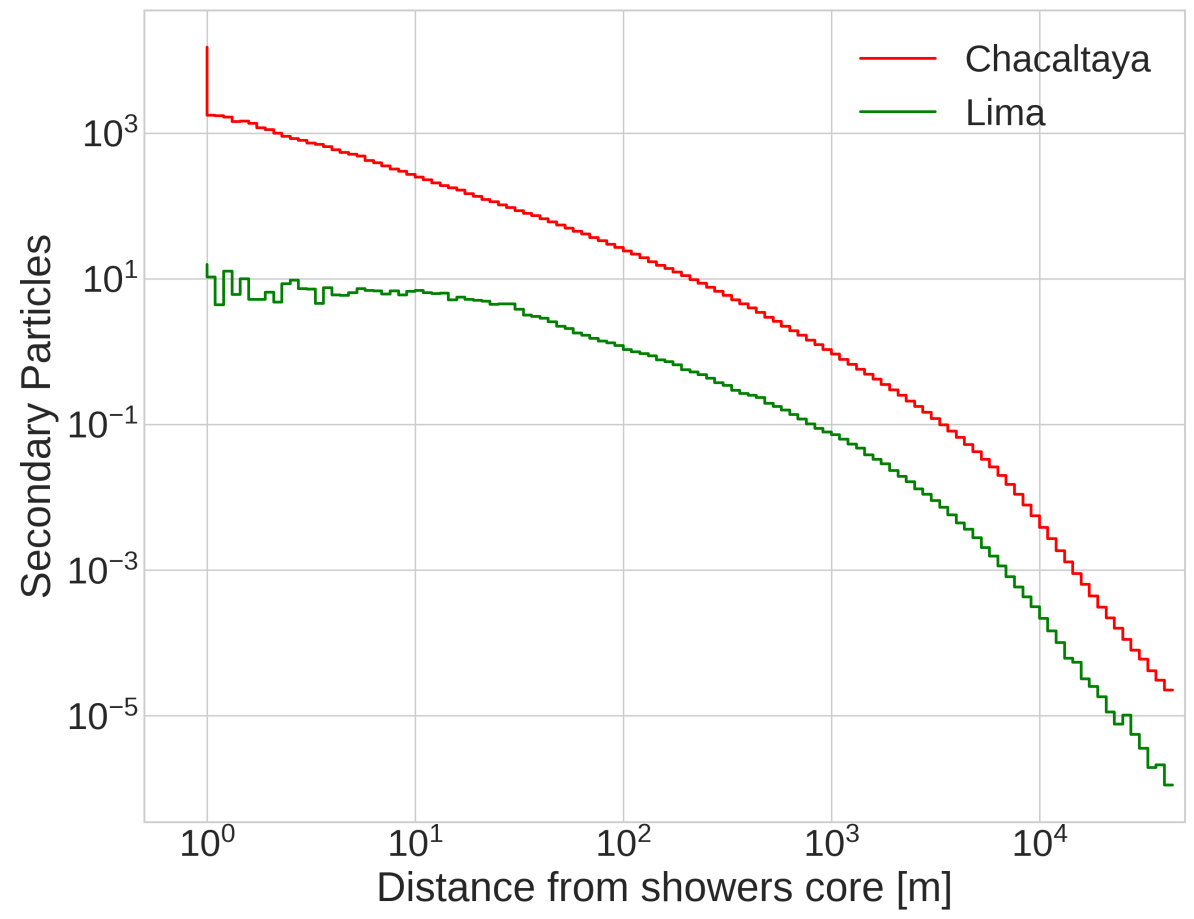

Figure 1: Density of particles as a function of their distance from the core of the shower for the simulation of 1 hour of primary particles arriving at $1 \mathrm{~m}^{2}$ at the top of the atmosphere within the energy interval: $10^{11}<\mathrm{E} / \mathrm{eV}<10^{15}$.

\section{Multiparticles indentification into the secondary flux at detector level}

According with the results presented in Fig. 1, and assuming an ideal detector with efficiency equal one, we expect a ratio $\sim 1000 \mathrm{~Hz}$ for secondary particles at detector level. On the other hand, if a particle crossing a LAGO's detector produces a signal that overcome a setting threshold, the electronic stores this signal during $300 \mathrm{~ns}$. Therefore, if two or more particles hits the ground in a time window of $300 \mathrm{~ns}$ we refer to them as multiparticles. In this sense, and as approximation, we are assuming that not more than one primary reaches the atmosphere at the same time, so in this work all the multiparticles are coming from the same primary.

To cuantify the number of multiparticles presents in one hour of simulated flux, we designed an algorithm to identify multiparticles at detector level for each shower. To perform this calculation, the ground has been segmented in squares of $4 \mathrm{~m}$ side, as an approximation of the real effective area of a WCD from the LAGO network [1,3], and then we search for multiparticles on each of this surfaces.

We have applied this algorithm for two LAGO sites: Chacaltaya, Bolivia (5200 m a.s.1.), and Lima, Perú, (950 m a.s.l.) to identify only electromagnetic multiparticles (i.e. $\mathrm{e}^{ \pm}$and $\gamma$, EM- 
multiparticles, hereafter). The results are presented in Fig. 2, where the spectra for the electromagnetic component it is showed in black for both sites, and the spectra of the identified multiparticles in red (Chacaltaya), Fig.2 left, and green (Lima), Fig.2 right. The atmospheric absorption effect on the EAS development is apreaciated as a reduction of the total flux to energies that extents up to $\sim 10 \mathrm{GeV}$ and the cutoff above this same energy at Lima. This effects are accorging our previous results [3].

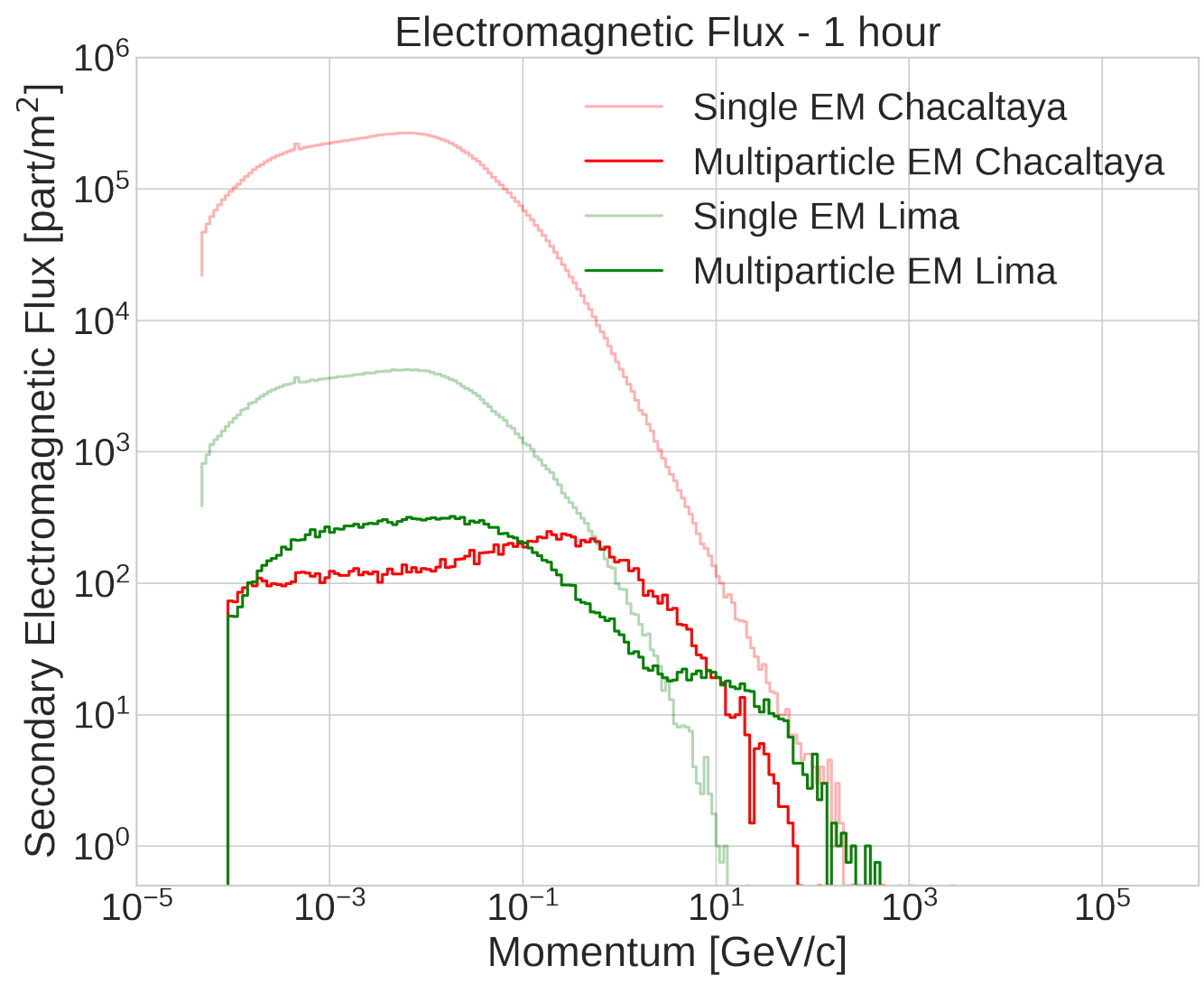

Figure 2: Electromagnetic component of secondary cosmic ray spectrum calculated by ARTI for the Chacaltaya and Lima sites. It is also showed the spectra of multiparticles that reach the detection area.

Fig. 3 shows the discrimination of electromagnetic, muonic and hadronic part of the EAS, simulated using ARTI, as they reach the gorund level at both sites, Chacaltaya and Lima. In order to show how the atmospheric absrotion affect each component, it can be seen that the electromagnetic and hadrons are strogly affected, and regarding muons, the harder portion of the spectrum reachs the ground at Lima, as expected. This comparison was performed to evaluate if the calculated multiparticle spectra influence the muon spectra in our calculations. As a result we see no ovelapping among the mentioned component of the EAS.

Regarding EM-multiparticles, at Chacaltaya it is possible to see a plateau for energies below $10^{-2} \mathrm{GeV}$ where the number of these particles is $\sim 0.01 \%$ of the single particles. Above this energy and until $\sim 2 \times 10^{-1} \mathrm{GeV}$ the EM-multiparticles increase as a power law respect to energy (log-log 

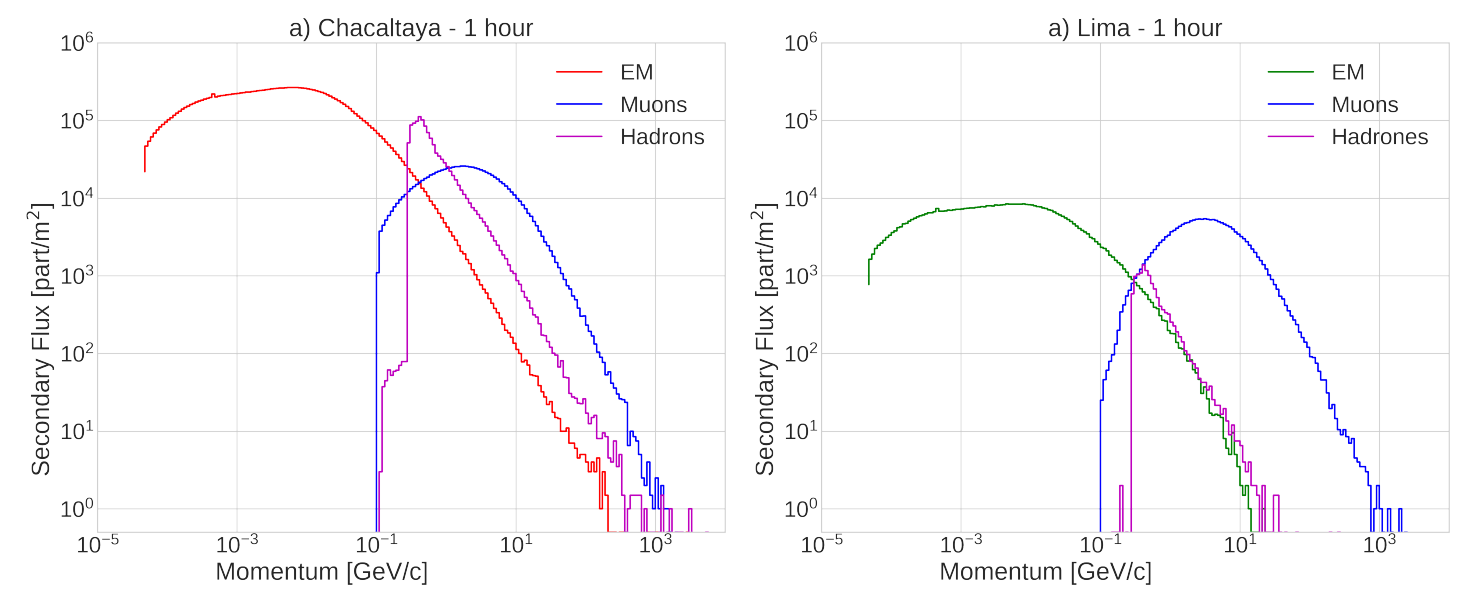

Figure 3: Electromagnetic, muonic and hadronic components of the secondary cosmic ray spectrum calculated by ARTI for the Chacaltaya (left) and Lima (rigth) sites.

scale) to start decreasing after this last energy, where the number of single particles have dropped around two orders of magnitud. At Lima, the number of EM-multiparticles grow up to $10^{-3} \mathrm{GeV}$ from where this number starts a kind of plateau with a flux around $10 \%$ respect the single particles, until $10^{-1} \mathrm{GeV}$. After here, this number decreases following a power law until $\sim 2 \mathrm{GeV}$ from where it starts a new plateau to $10^{1} \mathrm{GeV}$, and then to start dropping again.

The number of particles in the electromagnetic shower is 32333279 (1064870) for Chacaltaya (Lima), and the number of multiparticules results in 27040 (70250). This hows that around $0.08 \%$ $(6.59 \%)$ of this multiparticles are not taken in to account in the simulation, meaning a substancial loss when the simulations are performed to account for the real amount of EAS particles that are measured in a WCD.

In Fig. 4 the spectra of primaries producing EM-particles is presented in comparison with total flux $\Phi$. The number of primaries producing EM-multiparticles, at Chacaltaya site, increase with the energy from $\sim 10 \mathrm{GeV}$ to get a plateau at $\sim 10^{2} \mathrm{GeV}$ to $10^{3} \mathrm{GeV}$, from where the variation is dominated by the low statistic; whereas at Lima site, the same number starts to increase from $\sim 3 \mathrm{GeV}$ to get a short plateau from $\sim 20 \mathrm{GeV}$ to $\sim 150 \mathrm{GeV}$, from here and forth this quantity starts to decrease.

\section{Discusion and Conclusions}

In this work, we have estimated the number of electromagnetic multiparticles at two sites that are part of the LAGO detection network with two differents altitudes (atmosphere depth), $5200 \mathrm{~m}$ a.s.l. ( $\sim 50 \mathrm{gr} / \mathrm{cm}^{2}$ ( for Chacaltaya, Bolivia and $950 \mathrm{~m}$ a.s.l. $\left(\sim 1000 \mathrm{gr} / \mathrm{cm}^{2}\right)$ for Lima, Perú.

At Chacaltaya, the number of EM-multiparticles corresponds to close the $0.1 \%$ of the total flux up to $\sim 10^{-2} \mathrm{GeV}$, energy at which the number of protons and other secundaries hadrons starts to influence the espectrum.

In the case of Lima, the multiparticles flux can extend the spectrum up to two orders of magnitude $\sim 10^{3} \mathrm{GeV}$, Fig. 2. This feature could be understood as the decay of the EAS's hadronic 


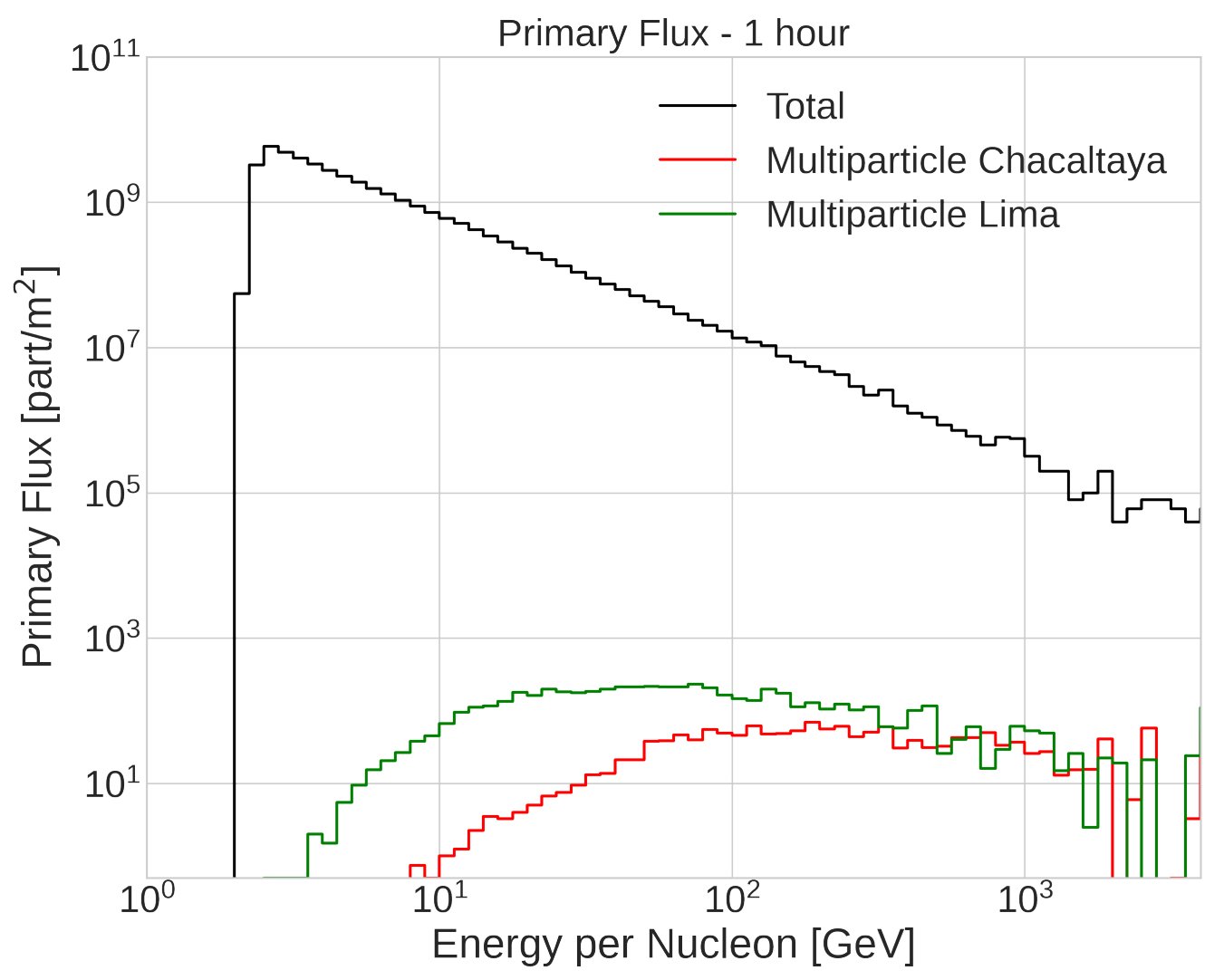

Figure 4: Energy spectrum per nucleon of primary cosmic rays that reach the top of the atmosphere, calculated in the ARTI framework and same spectrum for the primaries that generate multiparticles on detection surface.

or muonic components, i.e. the hadrons and muons have enough time to decay and produce more particles, as it can see in Fig. 3.

Since the LAGO calibration uses the vertical muon equivalent (VEM) technique, that in LAGO detectors has a peak at $200 \mathrm{MeV}$, we have noted that the extended multiparticle spectrum coincides with the expected muon spectrum, Fig. 3. So, an influence on the calibration is expected.

Finally, we also note that the multiparticles spectra can give us information about the primary spectra, in the $10 \mathrm{GeV}-1 \mathrm{TeV}$ range, as can be seen in Fig. 4.

The algorithm for search multiparicles can be implemented in a future version of LAGO's ARTI simulation framework. A more profound study must be done in order to fully understad the relation between primaries and multiparticles in different altitude conditions.

\section{Acknowledgements}

CSC, IS and HA acknowledge the support of CONICET and CNEA. This work has been partially carried out on the ITeDA cluster, we thank A.P.J. Sedoski Croce for his continuous support. The LAGO Collaboration is very thankful to all the participating institutions and to the Pierre Auger Collaboration for their continuous support. 


\section{References}

[1] LAGO collaboration, The Sites of the Latin American Giant Observatory, in 34th ICRC, (The Hague, The Netherlands), p. PoS(ICRC2015)665, 2016, DOI.

[2] A. J. Rubio-Montero, R. Pagán-Muñoz, R. Mayo-García, A. Pardo-Diaz, I. Sidelnik and H. Asorey, The EOSC-Synergy cloud services implementation for the Latin American Giant Observatory (LAGO), (This proceedings), 2021.

[3] LAGO collaboration, Performance of the LAGO water Cherenkov detectors to cosmic ray flux, 2010.14591.

[4] H. Asorey, L.A. Núñez and M. Suárez-Durán, Preliminary results from the latin american giant observatory space weather simulation chain, Space Weather 16 (2018) 461 [https: //agupubs. onlinelibrary.wiley.com/doi/pdf/10.1002/2017SW001774].

[5] U.S. Atmosphere, US standard atmosphere, National Oceanic and Atmospheric Administration (1976).

[6] A. Letessier-Selvon and T. Stanev, Ultrahigh energy cosmic rays, Rev. Mod. Phys. 83 (2011) 907.

[7] B. Wiebel-Sooth, P.L. Biermann and H. Meyer, Cosmic rays. vii. individual element spectra: prediction and data, Astronomy and Astrophysics 330 (1998) 389.

[8] D. Heck, J. Knapp, J. Capdevielle, G. Schatz, T. Thouw et al., Corsika: A monte carlo code to simulate extensive air showers, Report fzka 6019 (1998). 


\section{Full Authors List: LAGO LAGO Collaboration}

V. Agosín ${ }^{20}$, A. Alberto ${ }^{3}$, C. Alvarez ${ }^{16}$, J. Araya ${ }^{20}$, R. Arceo ${ }^{16}$, O. Areso ${ }^{13}$, L. H. Arnaldi ${ }^{2}$, H. Asorey ${ }^{14,7}$, M. Audelo ${ }^{9}$, M.G. Ballina-Escobar ${ }^{19}$, D. C. Becerra-Villamizar ${ }^{18}$, X. Bertou ${ }^{2}$, K.S. Caballero-Mora ${ }^{16}$, R. Caiza ${ }^{8}$, R. Calderón-Ardila ${ }^{14}$, Calle, J. ${ }^{24}$, A. C. Fauth $^{27}$, E. Carrera Jarrin ${ }^{26}$, L. E. Castillo Delacroix ${ }^{11}$, C. Castromonte ${ }^{25}$, Cazar-Ramírez D. ${ }^{26}$, Diego Cogollo ${ }^{28}$, D. A. Coloma Borja $^{26}$, R. Conde ${ }^{1}$, J. Cotzomi ${ }^{1}$, D. Dallara ${ }^{11}$, S. Dasso ${ }^{13,5,6}$, R. Aguiar ${ }^{27}$, Albuquerque, A. ${ }^{28}$, J.H.A.P.Reis ${ }^{27}$, H. De León ${ }^{16}$, R. deLeón-Barrios ${ }^{23}$, D. Domínguez ${ }^{8}$, M. Echiburu ${ }^{21}$, M. González ${ }^{2}$, M. Gómez Berisso ${ }^{2}$, J. Grisales Casadiegos ${ }^{23}$, A. M. Gulisano ${ }^{13,12,6}$, Juan Carlos Helo ${ }^{17}$, Condori, C. A. H. ${ }^{24}$, J. E. Ise ${ }^{11}$, Nascimento, G. K. ${ }^{28}$, M. A. Leigui de Oliveira ${ }^{29}$, F. L. Miletto ${ }^{27}$, V. P. Luzio ${ }^{29}$, F. Machado ${ }^{25}$, Juan F. Mancilla-Caceres ${ }^{22}$, D. Manriquez ${ }^{20}$, A. Martínez-Méndez ${ }^{23}$, O. Martinez ${ }^{1}$, R. Mayo-García ${ }^{3}$, L.G. Mijangos ${ }^{22}$, Miranda, $\mathrm{P}^{24}$, M. G. Molina ${ }^{11}$, I.R. Morales ${ }^{19}$, O.G Morales-Olivares ${ }^{16}$, E. Moreno-Barbosa ${ }^{1}$, P. Muñoz ${ }^{17}$, Nina, $\mathrm{C}^{24}$, L.A. Núñez ${ }^{23}$, L. Otininano ${ }^{4}$, R.Pagán-Muñoz ${ }^{3}$, K. M. Parada-Jaime ${ }^{18}$, H. M. Parada-Villamizar ${ }^{18}$, R. Parra ${ }^{10}$, J. Peña-Rodríguez ${ }^{23}$, M. Pereira ${ }^{13}$, Y. A. Perez-Cuevas ${ }^{18}$, H. Perez ${ }^{19}$, J. Pisco-Guabave ${ }^{23}$, Raljevic, M. ${ }^{24}$, M. Ramelli ${ }^{13}$, C. Ramírez ${ }^{22}$, Rivera, ${ }^{24}$, L. T. Rubinstein ${ }^{13}$, A.J. Rubio-Montero $^{3}$, J.R. Sacahui ${ }^{19}$, H. Salazar ${ }^{1}$, N. Salomón ${ }^{11}$, J. Samanes ${ }^{4}$, N.A. Santos ${ }^{5}$, C. Sarmiento-Cano ${ }^{14}$, I. Sidelnik ${ }^{2}$, Mayra B. Silva $^{22}$, O. Soto ${ }^{17}$, M. Suárez-Durán ${ }^{18,31}$, Subieta Vasquez, M. ${ }^{24}$, Terrazas C. ${ }^{24}$, Ticona, R. ${ }^{24}$, T. Torres Peralta ${ }^{11}$, Pablo A. Ulloa ${ }^{17}$, Z.R. Urrutia ${ }^{22}$, N. Vásquez ${ }^{8}$, A. Vázquez-Ramírez ${ }^{23}$, A. Vega ${ }^{20}$, P. Vega ${ }^{17}$, J. Vega $^{4}$, A. Vesga-Ramirez ${ }^{14}$, D. Vitoreti ${ }^{30}$, R. Wiklich Sobrinho ${ }^{29}$,

${ }^{1}$ Benemérita Universidad Autónoma de Puebla". ${ }^{2}$ Centro Atómico Bariloche (CNEA/CONICET/IB)". ${ }^{3}$ CIEMAT". ${ }^{4}$ Comisión Nacional de Investigación y Desarrollo Aeroespacial". ${ }^{5}$ Departamento de Ciencias de la Atmósfera y los Océanos, Facultad de Ciencias Exactas y Naturales, Universidad de Buenos Aires.". ${ }^{6}$ Departamento de Física (FCEN,UBA)". ${ }^{7}$ Departamento Física Médica, CNEA-CONICET-UNSAM". ${ }^{8}$ Escuela Politécnica Nacional". ${ }^{9}$ Escuela Superior Politécnica de Chimborazo". ${ }^{10}$ European Soutern Observatory (ESO)". ${ }^{11}$ Facultad de Ciencias Exactas y Tecnología (FACET) - Universidad Nacional de Tucumán (UNT)". ${ }^{12}$ Instituto Antártico Argentino, Dirección Nacional del Antartico, Instituto de Astronomía y Física del Espacio (UBA-CONICET)". ${ }^{13}$ Instituto de Astronomía y Física del Espacio, IAFE (UBA-CONICET)". ${ }^{14}$ Instituto de Tecnologías en Detección y Astropartículas (CNEA, CONICET,UNSAM)". ${ }^{16}$ Universidad Autónoma de Chiapas". ${ }^{17}$ Universidad de La Serena". ${ }^{18}$ Universidad de Pamplona". ${ }^{19}$ Universidad de San Carlos". ${ }^{20}$ Universidad de Valparaíso". ${ }^{21}$ Universidad de Viña del Mar". ${ }^{22}$ Universidad del Valle de Guatemala". ${ }^{23}$ Universidad Industrial de Santander". ${ }^{24}$ Universidad Mayor de San Andrés". ${ }^{25}$ Universidad Nacional de Ingeniería". ${ }^{26}$ Universidad San Francisco de Quito". ${ }^{27}$ Universidade Estadual de Campinas". ${ }^{28}$ Universidade Federal de Campina Grande". ${ }^{29}$ Universidade Federal do ABC". ${ }^{30}$ Universidade Federal do Recôncavo da Bahia". ${ }^{31}$ Université Libre de Bruxelles, Brussels, Belgium.". 